abnormality in heart conduct system. The patient in this case is a 15-year-old male with $17 \mathrm{~mm}$ perimembranous ventricular septal defect (PVSD) accompanied with critical pulmonary valve stenosis (PS) and II II auriculo-ventricular block (AVB). Having denied prophylactic permanent pacemaker implantation and open chest operation repair, this patient later was performed percutaneous balloon pulmonary valvuloplasty (PBPV) and subsequently transcatheter closure of VSD with a special designed $24 \mathrm{~mm}$ modified Amplatzer perimembranous VSD occluder without obvious residual intracardiac shunting and residual pulmonary valve stenosis (after 2nd stage PBPV). Transient complete heart block and junctional escape rhythm were developed one day after procedure and recovered 7 days later. During 4-year follow-up, no sequela was revealed by regular and ambulatory ECG monitoring. Placement of device confirmed satisfactory and no residual intracardiac shunting or heart valves regurgitation was detected echocardiographically. We deduced that the II II AVB might be congential and stable in this case. In our opinion, transcatheter closure of large VSD $(>15 \mathrm{~mm})$ and/or obviously ECG abnormality in heart conduct system appears to be an alternative option for carefully selected patients who are not willing to undergo surgical repairs. However, prognosis should be strictly evaluated by long time and multi-centre follow-up.

\section{e0539 B-TYPE NATRIURETIC PEPTIDE ON PREVENTING OF CONTRAST-INDUCED NEPHROPATHY IN PATIENTS WITH HEART FAILURE UNDERGOING PRIMARY PERCUTANEOUS CORONARY INTERVENTION}

doi:10.1136/hrt.2010.208967.539

\begin{abstract}
${ }^{1}$ Jing Zhang, ${ }^{2}$ Xianghua Fu, ${ }^{1}$ Oingsheng Wang, ${ }^{1}$ Xinna Fan, ${ }^{2}$ Yanbo Wang, ${ }^{2}$ Xuechao Wang. ${ }^{1}$ The No. 1 Hospital of Qinhuangdao; ${ }^{2}$ The Second Hospital of Hebei Medical University
\end{abstract}

Background Contrast-induced nephropathy (CIN) is one of the leading causes of hospital-acquired renal failure and increase in the mortality and length of hospital stay after percutaneous coronary intervention (PCI).

Purpose To evaluate the protective effect of B-type natriuretic peptide (BNP) on CIN in patients with heart failure undergoing PCI. Material and methods In the prospective, placebo-controlled, randomised trial, 149 consecutive acute myocardial infarction (AMI) patients with heart failure undergoing primary $\mathrm{PCI}$ received recombinant human $\mathrm{BNP}$ or placebo from the time of admission to $24 \mathrm{~h}$ after PCI. Serum creatinine (SCr) levels were measured to evaluate the protective effect of rhBNP on renal function. Estimated glomerular filtration rate (eGFR) was calculated by simplified modification of diet in renal disease study equation. CIN was defined as a postprocedure peak increase in serum creatinine $(\mathrm{SCr})$ of $>0.5 \mathrm{mg} / \mathrm{dl}$ or $>25 \%$ from baseline.

Results The baseline characteristics, including baseline demographics and clinical characteristics and angiographic and procedural features, were similar between the two groups. The SCr significantly increased after PCI, with the peak value at the 48th hour, and then began to decrease. Repeated measure ANOVA showed that the $\mathrm{SCr}$ after PCI was lower in the BNP group than that in the control group $(\mathrm{F}=5.056, \mathrm{p}=0.026$ ). At 24,48 (the peak value), and $72 \mathrm{~h}$ and 7 days after PCI the SCr was lower in the BNP group than that in the control group. At 7 days after PCI, the SCr showed a lower trend to the baseline level in the BNP group (88.42 \pm 15.02 vs $90.89 \pm 17.64 \mu \mathrm{mol} / \mathrm{L}, \mathrm{p}=0.120$ ), while it failed to do so in the control group $(96.63 \pm 17.26$ vs $90.44 \pm 15.37 \mu \mathrm{mol} / \mathrm{L}, \mathrm{p}<0.001)$. The eGFR significantly decreased after PCI, with the lowest value at $48 \mathrm{~h}$, and then it began to increase. The eGFR after PCI was higher in the BNP group than that in the control group $(\mathrm{F}=5.831, \mathrm{p}=0.017)$. At 7 days, eGFR showed a trend towards higher than the baseline level in the
BNP group (75.32 \pm 12.34 vs $73.42 \pm 14.86, p=0.120)$, while it failed to do so in the control group. At 48 and $72 \mathrm{~h}$ and 7 days after PCI, the eGFR in the BNP group was significantly higher than that in the control group. The occurrence of CIN was significantly lower in the rhBNP group than that in the control group (12 vs 24 cases, $\mathrm{p}=0.024$ ).

Conclusion Periprocedural use of BNP could further promote the recovery of renal function and decrease the occurrence of CIN compared with routine treatment alone in patients with heart failure undergoing primary $\mathrm{PCI}$.

\section{e0540 EFFICACY AND SAFETY OF TIROFIBAN-ASSISTED DELAYED PCI IN PATIENTS WITH ST-SEGMENT ELAVATION MYOCARDIAL INFARCTION}

doi:10.1136/hrt.2010.208967.540

Jia Xinwei, Gu Xinshun, Guo Xiaoping, Zhang Jing, Fan Weize, Hao Guozhen, Fu Xianghua. Second Hospital of Hebei Medical University

Objective To compare the outcomes of IIb/IIIa antagonist assisted PCI within $12-72 \mathrm{~h}$ of onset with that of selective PCI within 7-10 days in STEMI patients

Methods Totally, 80 patients were randomly allocated into the delayed PCI group $(n=38)$ and the selective PCI group $(n=42)$. In the delayed PCI group, PCI was performed within 12-72 h of onset. Tirofiban $(10 \mu \mathrm{g} / \mathrm{kg})$ was administered intravenously over $3 \mathrm{~min}$ immediately before PCI, and then was intravenously administered at $0.15 \mu \mathrm{g} / \mathrm{kg} / \mathrm{min}$ during the procedure and for at least $36 \mathrm{~h}$ after PCI. In selective PCI group, PCI was performed within 7-10 days of onset. Blood platelet aggregation rate (PAR) was measured immediately before PCI (time 0 ) and at sequently different time points (30 min, $2 \mathrm{~h}, 6 \mathrm{~h}, 12 \mathrm{~h}, 48 \mathrm{~h}$ and 7 days). Final TIMI grade flow (TGF), corrected TIMI frame count (CTFC) and TIMI myocardial perfusion grade (TMPG) of the infarction related artery were recorded. Ultrasonic cardiography (1 week and 12 week after PCI) and raidonuclear ventricle imaging (RNVI) (1 week after PCI) parameters such as left ventricular peak ejection rate (LPER), left peak filling rate (LPFR), left ventricular time to peak ejection rate (LTPER) and left ventricular time to peak filling rate (LTPFR) were performed. Bleeding complications and major adverse cardiac events (MACE) were followed up for 3 months.

Results In delayed PCI group, the cases with TGF 0-1 were significantly fewer before PCI, while the cases with TGF 3, TMPG 3 were more and CTFC was lower after PCI. The LPER, LPFR 1 week after PCI in delayed PCI group was higher and the LTPER, LTPFR were lower. LVEDD at 1 week and 3 months after PCI was all significantly smaller, while the LVEF was higher. There were no significant difference between the two groups regarding the incidence of hemorrhagic complications and MACEs.

Conclusion Tirofiban facilitated delayed PCI for patients with STEMI of over $12 \mathrm{~h}$ of symptom onset is safe and effective.

\section{e0541 PERCUTANEOUS VALVED STENT IMPLANTATION ABOVE THE CORONARY OSTIA: A NEW TRANSITIONAL TREATMENT FOR ACUTE AORTIC VALVE RUPTURE}

doi:10.1136/hrt.2010.208967.541

${ }^{1}$ Zong Gang-jun, ${ }^{2}$ Bai Yuan, ${ }^{1}$ Wu Gang-yong, ${ }^{1}$ Xia Yan, ${ }^{2}$ Oin Yong-wen. ${ }^{1}$ Departments of Cardiology*, 101 Hospital of Pla, Wuxi, Jiangsu Province, China; ${ }^{2}$ Departments of Cardiology, Changhai Hospital, Second Military Medical University, Shanghai, China

Objective To investigate the feasibility of percutaneous valved stent implantation above the coronary ostia as a transitional treatment for acute aortic valve rupture.

Background In recent years, some experimental and clinical studies about percutaneous aortic valve replacement has been conducted. Under current conditions, the risk of this technique is still high. 
aortic valved stent implantation above the coronary ostia might avoid blocking the coronary ostia.

Method Fresh pig pericardia were procured and processed to make artificial valves, and then mounted on tubular braided stents to make valved stents. Twenty healthy dogs weighing $17.7 \pm 3.1 \mathrm{~kg}$ were selected to establish a canine model of acute aortic valve rupture. The dogs were randomly divided into two groups: the rupture group without any treatment and the valved stent group with percutaneous valved stent implantation above the coronary ostia. The two groups of animals were followed up for 3 months. Echocardiography and other tests were performed to assess aortic regurgitation and ventricular function.

Results Acute aortic valve rupture models were successfully established in 16 of 20 dogs. In the rupture group, the mean aortic regurgitation was $6.8 \pm 1.9 \mathrm{ml} / \mathrm{s}$, only three of eight animals survived for 3 months. In the valved stent group, the mean aortic regurgitation was $7.0 \pm 2.1 \mathrm{ml} / \mathrm{s}$, valved stents were successfully implanted above the coronary ostia in eight animals. Instant postimplantation anatomy showed that the stents were located appropriately. Seven dogs survived for 3 months. Reduced aortic regurgitation was detected by colour Doppler echocardiography and no valved stent dislocation was found by 64 -slice CT scan.

Conclusion Percutaneous valved stent implantation above the coronary ostia is feasible and effective as a transitional treatment for acute aortic valve rupture.

\section{e0542 TRANSCATHETER THERAPY OF VENTRICULAR SEPTAL RUPTURE IN PATIENTS WITH ACUTE MYOCARDIAL INFARCTION COMPLICATED BY VENTRICULAR SEPTAL RUPTURE}

doi:10.1136/hrt.2010.208967.542

WANG Guang-Yi, HAN Bao-Shi, WANG Zhi-Feng, HOU Hai-Jun, LI Yue, WANG Yu, ZHI Guang, XU Yong, CHEN Yun-Dai. Department of Cardiology, Chinese PLA General Hospital

Objective To explore the feasibility and clinical effects by transcatheter interventions used for the patient suffering acute myocardial infarction (AMI) complicating with ventricular septal rupture (VSR) postinfarction.

Methods Between June 2005 and August 2009, Ventricular septal rupture (VSR) patients diagnosed with VSR underwent transcatheter closure aged 59-79years old (mean 63.3 \pm 24.7 ). The occluders were released with the monitoring of transthoracic echocardiography (TTE) and X-ray fluoroscopy.

Results 3 cases were successfully treated with transcatheter closure. The defect diameter was 11-28 (17 \pm 9.5$) \mathrm{mm}$ measured by TTE and the size of the occluder was $16-32(21.3 \pm 7.6) \mathrm{mm}$. The occluder successful rate was $100 \%$. The case success in 2 and a big VSR lead to die in 1.

Conclusions VSR was showed by praecordial area auscultation, transthoracic echocardiography (TTE) and left ventricular angiography after AMI. The transcatheter closure of VSR postinfarction is alternative method in patients not suite for surgical closure. But the careful examination and preparation are need before transcatheter closure.

\section{E0543 CLINICAL AND CORONARY ANGIOGRAPHY CHARACTERISTICS BETWEEN YOUNG (<45) AND OLD (>60) PATIENTS WITH CORONARY ARTERY DISEASE}

doi:10.1136/hrt.2010.208967.543

Zhang Weiling, Oin Zhongsheng, Pan Sancong, Wan li, Liu Ying, Cui Huahua, Zhang Haiqin, Sun Yuxia, Li Xiangbin. Jincheng People's Hospital Department of Cardiology

Objective To study the clinical Clinical and coronary angiography characteristics between young $(\leq 45)$ and old $(>60)$ patients with coronary artery disease.
Methods Angiographic and clinical data from A: 176 patients $(\leq 45)$ selected from 1795 patients with coronary artery disease from April 2006 to May 2010 were compared to B: 464 patients (>60) with coronary artery disease from April 2007 to May 2009 in our department.

Results (1) The male in A much more than B $(93.1 \%$ vs $61.4 \%$, $\mathrm{p}<0.01)$. (2) The patients with hypertension or type 2 diabetes mellitus in A were less than B (all $p<0.01)$. (3) The patients with Smoking, taking drug named Anajia or a positive family history in coronary artery disease in A were much more than $B($ all $p<0.01$ ). The incidences of dyslipidemia in A were more than B $(28.2 \%$ vs $19.0 \%, p<0.05)$. (4) The patients because of acute myocardial infarction come to hospital were more than B $(72.0 \%$ vs $62.5 \%$, $\mathrm{p}<0.01$ ). (5) Auto driver, the self-employed and government functionary were the top three categories of the coronary heart disease, but farmer, worker and retired military cadre in B. (6) Morbidity: the patients $(\leq 45)$ with coronary artery disease accounted for $9.7 \%$ in all patients with coronary artery disease in the same time (the cases 82 ( $\leq 40)$ accounted for 4.6\%). (7) The patients done coronary angiography in A were more than B $(64.0 \%$ vs $38.1 \%, \mathrm{p}<0.01)$. Singer vessel coronary artery diseases were seen more frequently in A than B $(50.9 \%$ vs $21.5 \%, p<0.01)$, and especially left anterior descending branch diseases occupied $87.7 \%$; two-vessel diseases and collateral circulation were less in A than $B(p<0.05)$; right coronary artery and circumflex diseases were less in $A$ than $B(p<0.01)$; however lesions and left main artery, left anterior descending branch diseases have no statistics meaning in $\mathrm{A}$ and $\mathrm{B}$. The meaningless lesion coronary arteries diseases were much more in $\mathrm{A}$ than $\mathrm{B}(89.0 \%$ vs $0.6 \%, p<0.01)$. The myocardial infarction patients account for $90 \%$ in the groups who had meaningless coronary arteries change showed by coronary angiography in A.

Conclusion The feature of coronary heart disease ( $\leq 45)$ : (1) Most because of acute myocardial infarction come to hospital; (2) The male, short of labour, more tension, heavy work pressure, the incidences of dyslipidemia, smoking and the early onset group had stronger family history of coronary arteries diseases were high risk groups. The patients taking Anajia may also the high risk groups in our area. (3) Morbidity: accounted for $9.7 \%$ in all patients with coronary artery disease in the same time (the cases $(\leq 40)$ accounted for $4.6 \%$ ). (4) The coronary angiography characteristics about half is the single vessel diseases, especially left anterior descending branch diseases; coronary angiography in some patients has no significant lesions, and mainly to myocardial infarction; there were differences about two and three-vessel diseases, right coronary artery and circumflex diseases, collateral circulation between young $(\leq 45)$ and old ( $>60)$; there were not differences about the left main coronary arteries diseases, left anterior descending branch diseases and the degree of narrowed coronary arteries between young $(\leq 45)$ and old $(>60)$.

\section{e0544 THE SAFETY AND EFFICIENCY OF DALTEPARIN DURING PERCUTANEOUS CORONARY INTERVENTION}

doi:10.1136/hrt.2010.208967.544

${ }^{1}$ Shi Xu-bo, ${ }^{1}$ Li Tian-chang, ${ }^{2}$ Wang Hong-yu, ${ }^{3}$ Sun Gen-yi, ${ }^{4}$ Chen Bu-xing,

${ }^{5}$ Wang Ming-sheng. ${ }^{1}$ Department of Cardiology, Tongren hospital, Capital University of Medical Science; ${ }^{2}$ Department of Cardiology, People's Hospital, Peking University; ${ }^{3}$ Department of cardiology, Tianjin Chest Hospital; ${ }^{4}$ Department of Cardiology, Beijing Electric Power Hospital; ${ }^{5}$ Department of Cardiology, Beijing Shijingshan Hospital

Objective This study was designed to evaluate the efficacy and safety of using dalteparin instead of UFH in selective percutaneous coronary intervention (PCI) of patients with coronary heart disease (CHD).

Methods In this prospective, open-label, multicentre trial, from Jan. 2006 to Apr. 2008, 212 patients with CHD were enrolled. Patients without prior SC dalteparin therapy or those for patients who came 